\title{
The Role of Artificial Intelligence in Mitigating Unknown- Unknown Risks
}

\author{
Fatma Beshwari ${ }^{\mathrm{a}}$, Maryam Beshwari ${ }^{\mathrm{a}}$, Ayesha Beshwari ${ }^{\mathrm{a}}$ \\ ${ }^{\mathrm{a}}$ f.beshwari@gmail.com \\ ${ }^{a}$ Project Management Graduate, Hamdan Bin Mohammed Smart University, Dubai, UAE
}

\begin{abstract}
Artificial intelligence (AI) is a potentially powerful tool to mitigate the unknown unknown risk. This research's objective is investigating the impact of $\mathrm{AI}$ on mitigating unknown unknown risk. For all six cases analyzed, it was possible to extract that unknown risk is hard to be reported in advance. Therefore, since the outbreak of the Coronavirus COVID-19, organizations have been eager to use $\mathrm{AI}$ in response to the consequences caused by this pandemic. As a result, AI played a significant role in rapid response, decision-making, early prediction, automated processes, and detection and tracking. This means AI has the potential to be used as a solution to mitigate the post event impacts and study the patterns for fut ure prediction.
\end{abstract}

Keywords: Artificial Intelligence; Machine Learning; Unknown risks; Uncertainty; Coronavirus; COVID-19.

\section{Introduction}

Risk management is the guide to protect against any uncertainty and risk. The concept of 'unknownunknown risk', which is the lack of awareness of the existence of a risk that in itself is unfamiliar, has gained increasing attention in recent years, especially with the emerging crisis such as the Fukushima nuclear dis as ter. In many cases, the unknown unknown risk disturbed businesses, people, and caused huge losses.

Over last few months, as coronavirus spread across the world, countries are trying to learn about the COVID-19 to figure out how to slowdown the spread of the virus and defeat it. It has been very difficult for business and health care systems to plan for the impact of COVID-19 due to the lack of his torical comparisons of such outbreaks of this level and severity. Countries struggled to act against this event due to the unknown nature of it and its consequence, which led many to follow the containment method to limit the spread of this virus and be able to focus on finding better solutions to react. Many new strategies were followed to decrease the impact of the unknown risk of this event such as school closure, traveling limitations, work from home, etc. However, some of these strategies resulted in long-term damage to the economy due to the uncertainty of the shape of this virus during each stage and how to deal with it, which put a lot of stress on the healthcare system. In response to this risk, science and technologies have been working together to contain the negative consequences and reduce the losses for businesses and economies.

Allowing the potential of AI application in reacting against unknown unknown risk can be an opportunity to mitigate the impact of such risk. What makes it a good solution is the ability to increase in computing processing power, the rise of big data, and the adoption of cloud computing. In addition, AI can adapt ambiguous or contradictory messages through visual perception, decision-making, and speech recognition as well as translating between languages. AI is coming across as one of the main technologies that are accelerating the decision-making process based on real time insights. 


\subsection{Research Problem}

Many papers were published regarding unknown unknown risk and how to deal and overcome the challenges posed by this type of risk. Unknown unknown risk is a type of risk that can be considered as an extremely dangerous risk because its possibility and consequences are undetermined. The danger of unknown unknown risk is that since it is unpredictable and no one is aware of it, it cannot be managed at its initial appearance that in some cases leads to dis aster or organization bankruptcy and massive losses. Therefore, this research will aim to study the ambiguity of managing and acting towards the unknown risk. Specifically, it will examine the contribution of AI in mitigating the unknown risk and the management of change when this risk occurs. The capability of this technology can play a significant role in lowering the chances of negative consequences. This study will be based on the COVID-19 pandemic. It has been chosen because it illustrated the importance of technology in allowing life and businesses to continue as usual under such circumstances.

This paper will be divided into five sections. Firstly, the introduction, followed by a literature review addressing the unknown unknown risk, and Artificial Intelligence. The third section will gather data from secondary sources about different uses of AI in combating unknown unknown risk and COVID-19 will be a case study. The fourth section will perform a qualitative and quantitative analysis for these case studies, and based on the outcome, it will answer the hypothesis of this paper and present the effectiveness of this technology in mitigating unknown unknown risk.

\section{Literature Review}

\subsection{Unknown Unknown Risks}

An unknown unknown can be defined as the state of being unaware that a particular risk exists. The repetitive unknown unknown is not a word mistake; it refers to unknown likelihood and unknown impact. Another research defined it as events considered to be impossible to find or imagine in advance (Kim, 2012). Many researches have explored how to understand the unknown unknown, but the challenge exists in the nature of the event. In some research, they related the unknown unknown risk to the blacks wan theory, which is an unpredictable event that is beyond what is normally expected of a situation and has potentially severe consequences (Nafday, 2009). This term came from an ancient saying that presumed black swans did not exist and it was developed by Nassim Nicholas (Nafday, 2009). This is because both share the condition of having a major impact, extreme infrequency, and the widespread insistence. It is described by some as an outlier because it lies outside the realm of regular expectation, since nothing in the past can predict its possibility (Hajikazemi, Ekambaram, Andersen, Zidane, 2016).

Sometimes, problems exist in people rather than the event itself and the major obstacle to address this type of event is the lack of capability to accept it, and people who cannot cope with unknown unknown will sometimes actively ignore it (Alles, 2009). Another challenge is to turn as many unknown unknowns to known known and being proactive in being exploring organizational environment. Not all possibilities can be thought of and planned, but there are ways to minimize delays and negative impact caused by unknown unknowns. The biggest takeaway is to become better equipped at identifying, predicting, and managing the risks related to these occurrences.

Des pite that risk management performs as forward looking, it is not possible to identify all risks in advance (Hillson, 2005). Unknown Risk cannot be managed proactively and usually in unknown risk events, a workaround approach is used to respond to unidentified risk. The approach is based on planning once the unknown risk occurs and managing it. It is hard to predict it, but possible to manage and minimize its negative impact once it has happened. 


\subsection{Artificial Intelligence and Machine Learning}

$\mathrm{AI}$, as we know it, is the short form of Artificial Intelligence. This is where the science and technology come to play, with multiple approaches, on where the advancement of science creates a paradigm of virtue for every sector in any industry to succeed (Zhang and Peng, 2019). The famous mathematician Alan Turing, changed the world forever by asking a simple question and that is, 'Can Machines Think'? With in -depth research, he was able to prove the main and fundamental goal of artificial intelligence. AI was created for a reason and that is basically to replicate the human intelligence in a machine (Graham-Cumming, 2012). In simple words, AI can be defined as the simulation of human intelligence in machines that are programmed to think like humans and mimic their activities. This term can also be used for any machine that demonstrates characters associated with a human mind such as learning and problem solving.

Moreover, Machine Leaming (ML) is a subdivision of AI and refers to the study of computer algorithms that allow computer programs to automatically improve through experience (Mitchell, 2008). It is one of the ways to achieve AI and functions on small to large datasets by inspecting and correlating between the data to find common pattem. It is very important to understand how to choose the correct algorithm type and its logistic progression in order to get a correct output and avoid any bias affecting the application. Several researchers found that using correct algorithms and tools can help in dealing with uncertainty projects and complex environments better. In addition, they believed that AI as a tool is better and more accurate than traditional tools (Martinez and Fernandez-Rodriguez, 2015).

AI was created for the entire basis and the future of all complex decision making and it has been extremely efficient with its evolution of machine learning. There are several types of AI where machines are being taught and knowledge is leamed. Machines are given the opportunities to analyze and leam data just like the human brain (Motoda and Yoshida, 1998). With technology as advanced as this, there has been significant progress in manufacturing, finance, healthcare and even retail, all with the help of artificial intelligence (Zhang and Peng, 2019).

\subsection{Why AI is trending now?}

Over the past decades, the field of AI has made great progress toward computerizing human reasoning and it breakthrough in a wide diversity of sectors from healthcare to transportation. AI techniques are used by many industries through exploiting data and optimizing business and production processes. It processes the data in ways that are more dynamic and create value with faster and more accurate decision that result in reduced operational costs and personalized cus tomer experience (FERMA, 2019). In addition, ML is ass is ting financial services companies with advanced fraud detection, and computer vision is optimizing offline retail. AI can assist with an unbroken supply chain and boost productivity. Artificial intelligence is not only known to improve lives but has managed to save lives as well. AI has assisted with creating personalized drug protocols for a better diagnosis, with robots even assisting in surgeries (HE, 2019). AI is helping the humankind to save the world by creating smart agriculture with efforts to solve global challenges (Motoda \& Yoshida, 1998).

\subsection{The role of AI in COVID-19}

COVID-19 is a global pandemic that was first identified in Wuhan city of China and exponentially started to spread across the world. This pandemic has changed the world significantly, not only for health care systems, but also for politics, education, economics, transportation, etc. Consider what makes COVID-19, as a crisis is its unknown nature as a virus and its consequences, as well as the speed at which it is spreading across 
the world. Although World Health Organization (WHO) and Centers for Disease Control and Prevention (CDC) have published instructions and regulations to reduce the risk of COVID-19 from spreading, it is still breaking out (World Health Organization, 2020). Many countries considered this epidemic as a normal flu and ignored all advice released by WHO and followed the approach of herd immunity, but unfortunately this method made it worsen. This approach results in exploding the number of infected cases that eventually led those countries to issue a 24 hours lockdown while preventing any type of gathering as well as closing their borders (Mayo Clinic, 2020). In response to this unknown risk, AI played an important role and it has been applied successfully in managing this outbreak.

Countries like Japan, Hong Kong, and South Korea combined lessons leamed from H1N1 and SARS with the AI powered capabilities to process the unknown risk in real time which helped them to build defense and response systems to mitigate losses associated with the COVID-19 epidemic (Rogers, 2020). This proves that AI can analyze the anatomy of past crisis and apply that intelligence to the precau tions that countries develop today as they prepare for the challenges of tomorrow. The truth is countries and organizations cannot predict the onset of the global pandemic. However, they can identify the telltale indications. AI can empower organizations to proactively spot growing unknown risk early and take decisive measures before massive loss occurs. Fortunately, AI and unsupervised machine leaming are capable of analyzing, processing, and deriving actionable insight fromall this data and can do so in real time.

\section{Conceptual framework and hypothesized relationships}

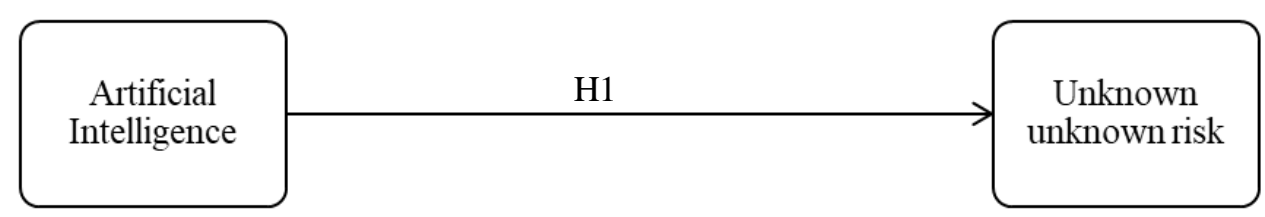

Fig 1. Research Conceptual Framework

Organizations are recognizing AI effects on the market and they became more interested in investigating $\mathrm{AI}$ as a new technology that can transform their management strategy. Unknown unknown risk is a big concern when it hurts the business without any contingency plan. Therefore, the ability of AI to predict and deal with the risk that might appear in the future is catching business attraction. Using AI to mitigate the upcoming unknown unknown risk in an organization can increase the chance of avoiding any loss. In addition, it will help the production to create new plans to reduce the risk. Moreover, they can manipulate it to their advantage, create an opportunity in having a change that exceeds that of opponents, and be the leader in the market. The objective of this research is to study AI capability towards unknown unknown risks negative consequences and the target audience for this research are organizations, project managers, and policy makers. Fig. 1 represents the proposed hy pothesis of this research. 


\section{Methodology}

After a complete review of the literature, a qualitative method has been decided to be used to meet the objective and answer the question of this study, which is how AI can mitigate the impact of unknown unknown risks. A combination of secondary data and interview methods will be utilized. As secondary data, six case studies of different utilizations of AI in combating COVID-19 will be chosen to discuss the role AI played in mitigating the unknown unknown risk. In addition, a structured interview will be used to gather detailed information and the questions will be based on an open-ended approach. This data will help to better understand in which stage risk management processes can involve AI technology and the potential level of its capabilities in unknown events cases.

\subsection{Data collection}

\subsubsection{Case A: Canada BlueDot}

The Canadian based AI model BlueDot that functions based on machine learning, predicts humans by spotting infectious disease outbreaks. It predicted the outbreak of the infection at the end of 2019 issuing a warning to its clients on 31 December 2019 before the WHO did so on 9 January 2020 (Kreuzhuber, 2020). A group of researchers who are working with the BlueDot listed the top 20-destination cities where passengers from Wuhan would arrive in the wake of the outbreak. They warned that these cities could be at the forefront of the global spread of the disease.

BlueDot aims not only to identify the threat of infectious diseases, but also to understand how diseases could spread to different parts of the world, then to determine the possible consequences of their spread. BlueDot was able not only to send out a waming, but also to correctly identify the cities that will be highly infected through using global air ticket data. It helped to predict where the infected people could potentially travel to. Eleven of the top listed cities were also the first areas affected by COVID-19 cases. For WHO, which is dependent on official statistics from Chinese authorities it was difficult to access such information and make forecasts early on (Phamet al., 2020).

\subsubsection{Case B: China Detection System}

CCTV based tracking was used in Asian countries to identify the potential infected people. China used infrared cameras to scan crowds for high temperature. Their AI powered system used facial recognition to identify individuals with high temperature and whether he/she is wearing mask. It is currently being used at Beijing's Qinghai Railway Station and it has the capability to inspect up to 200 people in one minute without interrupting passenger flow (Allam, Dey and Jones, 2020). This system also used to ensure citizens comply with self-quarantine instructions. According to reports, individuals who ignored the instruction and left home would get a call from the authorities after being tracked by the system (Preethika et al., 2020).

\subsubsection{Case C: Dubai Police Movementpermission}

The Dubai government announced a 24-hour sterilization program, which aims to reduce the physical contact between people and speed up the sterilization program. Dubai Police used AI to find if the trip was for the reason claimed in the permit request. AI was used along with speed radars to identify which vehicle has a movement permit or belongs to people working for important sectors. In addition, if a vehicle did not get a 
permit and went out for necessary purposes, AI can recognize the route and check if this trip was in fact for an important matter. Plus, the system has the capability to identify the path a person takes when they work in vital sectors and it will know the route fromhome to work.

\subsubsection{Case D: IBM Watson Assistant}

Watson Assistant is a convers ational AI platform that provides an engaging experience for users to get even their most complex issues solved by using supported chat and speech interfaces. It was preprogrammed with COVID-19 questions via the IBM cloud. Organizations in 12 countries from the city of Austin to the Polish Ministry of Health have been able to get up and running in as little as 24 hours (IBM, 2020). The objective of this type of technologies is to help reduce the waiting time for calls that users make to the telephone lines of state services relating to the novel coronavirus. In addition, it provides data driven insights to help employers make informed decisions on workplace re-entry, facilities management, space allocation, and other covered related priorities

\subsubsection{Case E: Microsoft Azure AI - immuneCODE}

Biotechnology and Microsoft collaborated in launch an immuneCODE that is an open database to share an immune response toward COVID-19 blood samples from patients around the world. The database contains information details on " $T$ " cells, which specifically recognize features of the virus with unprecedented scale and speed. This cell has trove of information that can provide one consis tent trackable measure of immune response. In addition, it can help in diagnosing and managing COVID-19 and provide accurate assessment of immunity. As a result, data from the immuneCODE will speed up vaccination development, provide better diagnose, and reduce the spread of the virus (Microsoft, 2020). According to Kevin Scott (2020), Microsoft is using similar supercomputing capacity that have been used before for natural language process to run molecular simulation to identify potential therapy for COVID-19.

\subsubsection{Case F: Facebook Fighting Misinformation}

Facebook uses AI model to detect and track false news written in text and images effectively. The software is trained to find duplicated or slightly modified version of that content as it spread across the platform in different languages (Statt, 2020). It can recognize and track both the copy and the original image with perhaps one changed word in the post. It can recognize whether the images contain false claims or misleading information about COVID-19 and apply warning label automatically (Statt, 2020). The system is applied to every image that has been uploaded on each platform, Instagram, and Facebook, which means billions of images are being checked per day in their database.

\subsection{Analysis}

\subsubsection{Case A:}

BlueDot was able to identify the crisis and the cities that will be affected by this disease that gives it the advantage of early prediction of such pandemic. It alerted the Canadian entity about this pandemic 8 day before the WHO announcement, which presents its effectiveness in early prediction (BlueDot, 2020). It also assisted in understanding how disease might scatter to different parts of the world and then determined the 
potential impacts. It pointed out which cities had the risk of transmitted COVID-19 despite having no official cases (BlueDot, 2020).

It required human interpretation and providing context to recognize the threat. In fact, even in the case of BlueDot, human remains central in evaluating and interpreting its output. Therefore, it is correct to stress that human input across dis ciplines is needed for the optimal application of AI.

\subsubsection{Case B:}

Case B used IOT devices as CCTV based on AI and machine leaming algorithm. It was able to detect infected people. In addition, it was used to track those who are not following the guidelines and report them. The data that was collected was interpreted and transferred to the concemed person in the organization for interpreting them, conducting results, and creating an action plan. Their main goal was to identify areas of vulnerability in early stages to avoid surprises by managing potential as pects, which can lead to occurrence of risk overtime. The shown AI system takes a whole CCTV as an input and runs a quick diagnosis to be able to provide the abnormal cases as an output.

\subsubsection{Case C:}

In Case C, they used AI to track people who got the permission in advance along with the ability of taking a decision regarding the fines, whether it is eligible or should be removed. The new understanding of the technology can be result in evaluating the current situation and making sense of a complex pattem of different as pects. Their aim was to produce a fast-reactive mechanis $m$ to various changes and pro-act to them. The effectiveness of their method was depending on the speed of analyzing the vehicle data. This system assisted in automating the validation processes, which in usual cases take days to be verified and can sometimes include human error (UAEGovernment, 2020).

\subsubsection{Case D:}

Case D used machine leaming to enable chatbots to interact with people and take decisions in responding to different inquiries from them later on. In addition, it automates the call center work to speed up the efficiency of this service and effectively carry out the process. The success of this mechanis m depends on the level of maturity of the organization in feeding up the basic knowledge and questions to the AI. As well as, expanding the sharing data for more than 12 countries for better proactive responding. Effectiveness and coverage are the two measures that provide the overall performance of Watson. With COVID-19, it succeeded because of its capability in responding to most inquiries well(Benvie, Wayneand Arnold, 2020).

\subsubsection{Case E:}

Azure ML empowers researches and medical organizations in responding to COVID-19 and it accelerated solutions to react against the global pandemic. The accuracy of the immune response will be continuously enhanced and updated online in real time as extra samples are sequenced by using MS Azure cloud and ML capabilities. The objective of this collaboration is to speed up the vaccination research and identify the patterns of this virus in timely manner. 


\subsubsection{Case F:}

Facebook AI system's goal is to reduce the spread of misleading information across the program. In addition, it helps them to demonstrate their commitment to keep Facebook and Instagram safe and genuine with the content uploaded in these platforms. It uses the three-pronged strategy of informing, reducing, and removing. It starts with informing users of recognizing false news. Then, it reduced the distribution and appearance of false news that has been labeled. Finally, it removes the fake posts and disable the accounts. According to Facebook Community Standards Enforcement Report (2020), the fake accounts represent approximately $5 \%$ of their users and were around 1.5 billion accounts in the second quarter of 2020. In addition, 99.6\% accounts were flagged with through their system before the other users reported them (Facebook Transparency Report|Community Standards, 2020).

After analyzing, we came up with five main tangible roles that AI played in COVID-19. Table 1 illustrates the identified influences in each case.

Table 1: Summary of AI contribution

\begin{tabular}{cccccc}
\hline Case & $\begin{array}{c}\text { Decision } \\
\text { Making }\end{array}$ & $\begin{array}{c}\text { Early } \\
\text { prediction }\end{array}$ & $\begin{array}{c}\text { Tracking and } \\
\text { detection }\end{array}$ & $\begin{array}{c}\text { Automation } \\
\text { processes }\end{array}$ & $\begin{array}{c}\text { Cost } \\
\text { efficiency }\end{array}$ \\
\hline Case A & $\bullet$ & $\bullet$ & $\bullet$ & $\bullet$ & $\bullet$ \\
\hline Case B & $\bullet$ & $\bullet$ & $\bullet$ & $\bullet$ & $\bullet$ \\
\hline Case C & $\bullet$ & $\bullet$ & $\bullet$ & $\bullet$ & $\bullet$ \\
\hline Case D & $\bullet$ & $\bullet$ & $\bullet$ & $\bullet$ & $\bullet$ \\
\hline Case E & $\bullet$ & $\bullet$ & & $\bullet$
\end{tabular}

\subsection{Effectiveness ofAI}

Table 2 addresses the six cases with using and without using AI for measuring the effectiveness of utilizing AI in COVID-19 and any future unknown events.

Table 2: measuring AI effectiveness - qualitative

\begin{tabular}{|c|c|c|}
\hline Case & Without AI & With AI \\
\hline Case A & $\begin{array}{l}\text { Human needs time and effort to analyze and find the } \\
\text { pattern to predict the pandemic as well as he will be } \\
\text { observing and waiting for other countries to report the } \\
\text { infected cases. WHO took } 9 \text { days after BlueDot results to } \\
\text { announce the pandemic. }\end{array}$ & $\begin{array}{l}\text { Predicted the pandemic before the WHO } \\
\text { announcem ent and reported the top } 20 \text { cities that will } \\
\text { have the virus thorough correlating between the } \\
\text { airlines' tickets and infected cases in city Wuhan. } 11 \\
\text { cities were correct from the list. }\end{array}$ \\
\hline Case B & $\begin{array}{l}\text { CCTV will not be able to detect and take actions against } \\
\text { any infected cas es and it will require human intervention } \\
\text { to verify the videos and report the violators. }\end{array}$ & $\begin{array}{l}\text { AI directly detected the infected cases once they } \\
\text { passed through the CCTV as well as identified the } \\
\text { violators who are not wearing masks. }\end{array}$ \\
\hline Case C & $\begin{array}{l}\text { Requires human review that will take more than a week to } \\
\text { verify each video recorded and map it to the permissions. }\end{array}$ & $\begin{array}{l}\text { AI could identify the violators within seconds and } \\
\text { take the decision to issue fines. }\end{array}$ \\
\hline Case D & $\begin{array}{l}\text { The customer service agent will take time to ask for the } \\
\text { answer and respond to the phone call inquiries and } \\
\text { sometimes the responsible person is not available to }\end{array}$ & $\begin{array}{l}\text { A direct response is available to any inquiries because } \\
\text { of the data that has been fed to the machine learning } \\
\text { and any unknown question will be reported to a }\end{array}$ \\
\hline
\end{tabular}


answer and that can take more than one day.

The process of analyzing the $\mathrm{T}$ cell will be very slow and it will require time and efforts to study all the samples and find the common pattern which in some cases requires

Case E months. Even sharing data will require many approvals and permissions. This will lead to put huge pressure on medical organizations which are al ready overwhelmed with infected cases.

The process of tracking the source of fake news requires times as well as it is very hard to identify the news if its true or fake. higher level as a quick reaction.

AI speed up the analyzing process because of the open public database to find the common attributes of the virus as well as it provided better diagnose. It helped to drive model in a day instead of months which is normally required using older technique.

AI speed up the process of verification and assisted in tracking the sources of fake news in seconds instead of weeks. In addition to its capability to automatically takes the decision to remove the news.

In addition, we did a quantitative measurement of using AI technology in respect to the following metrics: speed, adoption, response, availability, and cost reduction. The scale of assessment will be from 1 to 5 where 5 presents excellent and 1 is low. The scores are based on the res earcher's point of views fromeach case.

Table 3: measuring AI effectiveness - quantitative

\begin{tabular}{rccccc}
\hline Case & Speed & Adoption & Response & Availability & Reduce cost \\
\hline Case A & 5 & 4 & 4 & 5 & 4 \\
\hline Case B & 4 & 4 & 4 & 5 & 4 \\
\hline Case C & 4 & 4 & 5 & 5 & 5 \\
\hline Case D & 4 & 4 & 3 & 5 & 4 \\
\hline Case E & 4 & 3 & 4 & 5 & 4 \\
\hline Case F & 4 & 4 & 5 & 5 & 3 \\
\hline
\end{tabular}

Table 3 illustrates that the five measurements reveal the effectiveness of utilizing AI in mitigating unknown risk. As shown in table 4 most of the scores were between 3 to 5 which indicate that AI has a strong potential in mitigating unknown unknown risks. AI based systems are always available and were not impacted by working from a distance and being on lockdown. AI quickly adapted the change with the pandemic and assisted in reducing the cost of operations that required human involvement such as responding to customer questions, monitoring people's movement, and checking the logs of violators. Moreover, it contributed in automation of many repetitive and manual processes that have been impacted by implementing working from a distance. In addition, AI participated in the better understanding of this unknown risk through analyzing the patterns and turning it to become known as well as provided recommendations on how to react in different stages of this disease.

\subsection{Results}

After analyzing the six case studies, the results that were obtained within the previously described method for each case of using AI technology can be summarized in the main roles of rapid response, namely, decision making, early prediction, automate processes, and detection and tracking. We can consider AI as a game changer for accelerating cognitive abilities and economic benefits. AI and machine learning can help proactively prepare for unknown unknown risk. In the COVID-19 case, we found that most companies have focused so far on reactive measures, but now it is the perfect time to take transformative action and be proactive. Companies that have already introduced AI should press ahead immediately in order to achieve maximum advantage in the near term. They should not hesitate to scale up because AI will be a significant 
level that can help them manage this crisis and future unknown unknown risk. The following are the main AI roles found in mitigating unknown unknown risk:

\subsubsection{Rapid Response}

During any pandemic, the role of technology rises and shines. For example, in COVID-19, AI and big data showed an extraordinary role in solving problems and increasing the potential for response to any future circumstances. It played a significant role in diagnosing coronavirus patients. Thus, AI enabled rapid and ongoing adaption and recognition to the new abnormal and can be open to change. It improves the adaptability required to address changing conditions at every step of the unknown unknown risks. It is able to process the combination of structured and unstructured data in a massive amount, which shows the patterns and results in decision-making.

\subsubsection{Decision Making}

$\mathrm{AI}$ is a decision support system that allows domain experts to generate diverse alternative scenarios of the future and imagine different possible outcomes including un likely, but potentially impactful future (Cotelle, et al., 2019). Any unknown risk has an impact on any business and the effect can be long-term, so utilizing technology that can think and learn is a great opportunity. The top management can benefit from the technology through better decision-making, providing great productive insights and visibility of risk that can be delivered by AI. The organizations that benefited from the AI vital role in fighting against COVID-19 will assist the future developments in their decision-making strategy. AI technology has the ability to use secondary data to provide reference suggestions when people are unsure or when there are multiple decisions (Jin et al., 2020).

\subsubsection{Improve Cost efficiency}

AI helps organizations to improve their work efficiency through reducing cost of the processes by automating day-to-day assistance and guidance in the risk management process (Cotelle, et al., 2019). For a small organization with few resources, AI can propose a method to achieve rapid and accurate risk assessment, which result in proper allocation of the limited resources. This means that AI led to substantial savings in term of human resources and improved emergency repair, efficiency, and decreased the risk of infection for individuals within the organization. The ability of adapting to different requirements can be considered as a benefit in the resources.

\subsubsection{Early Prediction}

Unknown risk has warning signs and if we can identify them and act against minor events, then perhaps we can avoid losses. It is important to obtain an early indication of negative risk development that will become noticeable in the future (Hajikazemi, Ekambaram, Andersen, Zidane, 2016). AI can be used to identify abnormalities in certain areas of data storage. With the huge amount of data, you would not see them as a person, whereas the machine now is enabling us to notice them. It has the potential to exceed humans, not only through speed, but also by dictating pattems, which humans have overlooked. These patterns will be taken under consideration and will generate many different scenarios exploring a variety of possible outcomes. The top management roles come to prepare the organization to deal with realistic circumstances for both expected and unexpected futures. 


\subsubsection{Automate Processes}

Automate repetitive tasks that a human does every day, but in doing so we are going to create work that will allow people to do more complex and judgment-based pieces of work that will enhance their ability to engage in the job. It conducts enterprise risk assess ment through identifying ris $\mathrm{k}$ materials in the organization, by calculating inherent risks using likelihood, impact, and analysis model. Second, it evaluates and redeems the risks by using context models to determine an action, such as accepting, monitoring, or treating. Then it performs residual risk analysis after controls are applied. Finally, it schedules risk assessment by running periodic risk assessment for analys is and evaluation. Not to mention, it has a self-learning feature which means the embedded self-learning feature of AI will keep learning for accurate results in the future (Christie, 2020). AI technology has shown good performance during the pandemic and can be used with different diagnos is levels, according to different policies that humans teach it. The system will keep leaming and reach to experienced levels, which later on will automatically produce the output of the matter (Jin et al., 2020).

\subsubsection{Detection and tracking}

Organizations need to analyze their risk profile through the competence of their risk management framework. The insufficient knowledge and the complete lack of awareness about their existence can make it hard on organizations to detect. This may lead to losses due to inadequate experience and interact ion with the available risk management system (Emerging). Detecting and tracking symptoms with AI can lower the possibility of losses and will lead to the creation of a relevant credible management model. This creates the opportunity to plan different scenarios and strategic foresight, which must go beyond the conventional approaches due to the emerging risk occurring from the complex and interrelated event.

For example, in the COVID-19 pandemic, AI technology was able to track the suspicious cases and forecast the nature of the virus from the feed up data about the risk infection and the likelihood of spreading. The monitoring feature resulted in predicting the possible positive cases and the regions in which they are likely to be present. Thus, it provided daily updated information, which aided in giving the possible measurements to prevent the virus from spreading accordingly.

\section{Limitation}

Many papers admitted that $\mathrm{AI}$ in some cases struggled due to lack of data. In fact, the successful implementation of artificial intelligence is dependent upon the data set and creativity of the humans who are deploying it to mitigate the unknown unknown risk. The value of AI and its application in exceptional times like the coronavirus pandemic stems from the human input that goes into its design process. To realize the full potential of AI, it is necessary to marry innovation and human action together, so that we can win the war against any type of unknown risk with reduced losses.

\section{Recommendations}

The following are recommend ations to enhance the role of AI in risk mitig ation:

- Governments must put in place the necessary supporting technology, regulations, and governance to enable large-scale adoption in response to unknown unknown risk.

- Promote co-operation, and data exchange both on a national scale and globally by the AI community, and policy makers to contribute in preparing for the problem, recognizing important data and launching datas ets for unknown unknown risk. 
- Dedicate research on AItechnologies that can understand fromlimited data.

- Build AI-powered monitoring tools that empower research without affecting privacy.

- Initiating emerging risk governance framework that contains new approaches to the risk management strategy, operational processes, and measurement of exposure to them.

- Integ ration AI with other technologies such as blockchain, big data, and cloud to achieve better efficiency in reacting to pre and post unknown unknown risk.

\section{Conclusion}

In conclusion, it is clearly seen that AI can be a game changer for mitigating unknown unknown risk. The capability of this technology, which was proven during the COVID-19 pandemic, is that it could be a valuable source to combat black s wan events. In this research, we have presented a state by utilizing AI technology to address numerous challenges posed by the coronavirus pandemic. Six main applications of AI potentials for coping with COVID-19 crisis have been analyzed all through. As a result, this research acknowledged that AI and machine-leaming technology are enabling the shift by providing the took to support remote communication, enable telemedicine, and protect food security. In addition to its capability of early prediction, tracking and detecting, decision-making, and process automation, we believe AI-driven algorithms could deliver valuable predictions and readings in the future. Companies do not require completely new processes for dealing with AI, but they will need to improve existing ones to consider AI and fill the necessary gaps. For best utilization, it is recommended to increase data sharing, in addition to having access to different historical data with the ability of pulling data from the right sources. However, the biggest challenge is to create a convincing and reliable global data set of different risks id entified among the nations with possible different metrics. Adoption of AI requires from companies to go through a learning journey and to work in a more scientific mind-set.

\section{References}

Clark, T., Woodley, R., De Halas, D., 1962. Gas-Graphite Systems, in "Nuclear Graphite" R. Nightingale, Editor. Academic Press, New York, p. 387.

Deal, B., Grove, A., 1965. General Relationship for the Thermal Oxidation of Silicon, Journal of Applied Physics 36, p. 3770.

Deep-Burn Project: Annual Report for 2009, Idaho National Laboratory, Sept. 2009.

Fachinger, J., den Exter, M., Grambow, B., Holgerson, S., Landesmann, C., Titov, M., Podruhzina, T., 2004.“Behavior of spent HTR fuel elements in aquatic phases of repository host rock formations," $2^{\text {nd }}$ International Topical Meeting on High Temperature Reactor Technology. Beijing, China, paper \#B08.

Fachinger, J., 2006. Behavior of HTR Fuel Elements in Aquatic Phases of Repository Host Rock Formations. Nuclear Engineering \& Design 236, p. 54

Alhashmi, S.F., Salloum, S.A. and Abdallah, S., 2019, October. Critical success factors for implementing artificial intelligence (AI) projects in Dubai government United Arab Emirates (UAE) health sector: Applying the extended technology acceptance model (T AM). In International Conference on Advanced Intelligent Systems and Informatics (pp. 393-405). Springer, Cham.

Allam, Z., Dey, G. and Jones, D., 2020. Artificial Intelligence (AI) Provided Early Detection of the Coronavirus (COVID-19) in China and Will Influence Future Urban Health Policy Internationally. AI, 1(2), pp.156-165.

Alles, M., 2009. Governance in the age of unknown unknowns. International Journal of Disclosure and Governance, 6(2), pp.85-88.

Baidu. 2020. How Baidu Is Bringing AI To The Fight Against Coronavirus. [onlinel Available at: <https://www.technologyreview.com/2020/03/11/905366/how-baidu-is-bringing-ai-to-the-fight-against-coronavirus/> [Accessed 27 June 20201.

Benvie, A., Wayne, E. and Arnold, M., 2020. Measure Watson Assistant Performance. [online] Available at: <https://www.ibm.com/downloads/cas/V0XQ0ZRE> [Accessed 20 July 2020].

Bloomberg Technology, 2020. How Microsoft Is Using AI To Find Covid-19 Antibodies, Says CTO. [video] Available at: $<$ https://www. youtube.com/watch?v=i0JYEDKypG0\&t=61s> [Accessed 8 July 2020].

BlueDot. 2020. Bluedot: Outbreak Risk Software. [online] Available at: <https://bluedot.global/> [Accessed 20 July 2020].

Chen, H., 2019. Success Factors Impacting Artificial Intelligence Adoption---Perspective From the Telecom Industry in China.

Cotelle, P., et al., 2019. Artificial Intelligence Applied To Risk Management. 3rd ed. [ebook] Brussels: Federation of European Risk 
Management Associations (FERMA). Available at: <https://www.ferma.eu/app/uploads/2019/11/FERMA-AI-applied-to-RMFINAL.pdf $\$$ [Accessed 10 July 2020].

FERMA., 2019. Artificial intelligence applied to risk management. [online] Available at: <https://www.eciia.eu/wpcontent/uploads/2019/11/FERMA-AI-applied-to-RM-FINAL.pd\$ [Accessed 15 September 2020].

Graham-Cumming, J., 2012. Alan Turing: Intelligence \& life. New Scientist, 214(2867), p.vi-vii.

HE, Y., 2019. The Importance of Artificial Intelligence to Economic Growth. Korean Artificial Intelligence, 7(1), pp.17-22.

Hajikazemi, S., Ekambaram, A., Andersen, B. and Zidane, Y., 2016. The Black S wan - Knowing the Unknown in Projects. Procedia Social and Behavioral Sciences, 226, pp.184-192.

Hillson, D., 2005. Why Some Risks Turn Into Surprises. [online] Available at: <https://www.projectmanagement.com/blogpost/24683/Why-some-risks-turn-into-surprises> [Accessed 28 June 2020].

IBM. 2020. COVID-19 Response Automation| Watson Assistant For Citizens. [online] Available at: <https://www.ibm.com/aeen/watson/covid-response> [Accessed 6 July 2020].

Jin, C., et al., 2020. Development and Evaluation of an AI System for COVID-19 Diagnosis.

Kim, D., 2012. Characterizing unknown unknowns. Paper presented at PMI® Global Congress 2012-North America, Vancouver, British Columbia, Canada. Newtown Square, PA: Project Management Institute.

Kreuzhuber, K., 2020. How AI, Big Data and Machine Learning can be used against the Corona virus. [online] Available at: < https://ars.electronica.art/aeblog/en/2020/03/19/ki-corona-part1/> [Accessed 01 July 2020]

Lassau, N., 2020. Key Success Factors for Successful Implementation of AI Based Segmentation Algorithms in Clinical Radiology Practice. American Journal of Biomedical Science \& Research, 7(3), pp.213-214.

Martínez, D.M. and Fernández-Rodríguez, J.C., 2015. Artificial Intelligence applied to project success: a literature review. IJIMAI, 3(5), pp.77-84.

Mayo Clinic. 2020. Herd Immunity And COVID-19 (Coronavirus): What You Need To Know. 「online] Available at: <https://www.mayoclinic.org/diseases-conditions/coronavirus/in-depth/herd-immunity-and-coronavirus/art-20486808> [Accessed 28 June 20201.

Microsoft. 2020. Adaptive Biotechnologies And Microsoft Launch Groundbreaking Immunecode Database To Share Populationwide Immune Response To The COVID-19 Virus - Stories. [onlinel Available at: <https://news.microsoft.com/2020/06/11/adaptivebiotechnologies-and-microsoft-launch-groundbre aking-immunecode-datab ase-to-share-populationwide-immune-respons e-to-thecovid-19-virus/> ‘Accessed 8 July 20201.

Motoda, H. and Yoshida, K., 1998. Machine learning techniques to make computers easier to use. Artificial Intelligence, 103(1-2), pp.295-321.

Nafday, A.M., 2009. Strategies for managing the consequences of black swan events. Leadership and Management in Engineering, 9(4), pp.191-197.

Pham, Q., Nguyen, D., Huynh-The, T., Hwang, W. and Pathirana, P., 2020. Artificial Intelligence (AI) and Big Data for Coronavirus (COVID-19) Pandemic: A Survey on the State-of-the-Arts. IEEE TRANSACTIONS ON ARTIFICIAL INTELLIGENCE, [online] Available at:

<https://www.researchgate.net/publication/340646512_Artificial_Intelligence_AI_and_Big_Data_for_Coronavirus_COVID19_Pandemic_A_Survey_on_the_State-of-the-Arts> [Accessed 26 June 2020].

Preethika, T., Vaishnavi, P., Agnishwar, J., Padmanathan, K., Umashankar, S., Annapoorani, S., Subash, M. and Aruloli, K., 2020. Artificial Intelligence and Drones to Combat COVID-19.

Rogers, A., 2020. Singapore Was Ready For Covid-19-Other Countries, Take Note. Ionlinel Available at: <https://www.wired.com/story/singapore-was-ready-for-covid-19-other-count ries-take-note/> [Accessed 26 June 2020].

Safdari, R., Saeedi, M.G., Valineiadi, A., Bouraghi, H. and Shahnavazi, H., 2017. Technology Acceptance Model in health care centers of Iran. International Journal of Computer Science and Network Security (IJCSNS), 17(1), p.42.

Statt, N., 2020. Facebook is using AI to combat COVID-19 misinformation and detect 'hateful memes'. The Verge, [online] Available at < https://www.theverge.com/2020/5/12/21254960/facebook-ai-moderation-covid-19-coron avirus-h ateful-mem es-hat e-speech> [Accessed 17 September 2020].

Spector, L., 2006. Evolution of artificial intelligence. Artificial Intelligence, 170(18), pp.1251-1253.

Facebook. 2020. Facebook Transparency Report | Community Standards. [online] Available at: <https://transparency.facebook.com/community-standards-enforcement\#fake-accounts> [Accessed 17 September 2020].

UAE Government. 2020. Movement Permits During Sanitisation Drive - The Official Portal Of The UAE Government. [online] Available at: $<$ https://u.ae/en/information-and-services/iustice-safety-and-the-law/handling-the-covid-19-outbreak/movem ent-permitsduring-sanitisation-drive> [Accessed 20 July 2020].

Vasant, P. and DeMarco, A. eds., 2015. Handbook of research on artificial intelligence techniques and algorithms. Information Science Reference.

Wixom, B.H. and Watson, H.J., 2001. An empirical investigation of the factors affecting data warehousing success. MIS quarterly, pp.1741.

World Health Organization. 2020. Coronavirus Disease (COVID-19) Pandemic. [onlinel Available at: <https://www.who.int/emergencies/diseases/novel-coronavirus-2019> [Accessed 26 June 2020].

Zhang, D. and Peng, G., 2019. Artificial Intelligence or Intelligence Augmentation? Unravelling the Debate through an Industry-Level Analysis. SSRN Electronic Journal. 